\title{
Genome-wide paternal uniparental disomy mosaicism in a woman with Beckwith-Wiedemann syndrome and ovarian steroid cell tumour
}

\author{
Magdalena Gogiel $^{1}$, Matthias Begemann ${ }^{1}$, Sabrina Spengler ${ }^{1}$, Lukas Soellner ${ }^{1}$, Ulf Göretzlehner ${ }^{2}$, \\ Thomas Eggermann ${ }^{\star 1}$ and Gertrud Strobl-Wildemann ${ }^{3}$
}

Uniparental disomy (UPD) of single chromosomes is a well-known molecular aberration in a group of congenital diseases commonly known as imprinting disorders (IDs). Whereas maternal and/or paternal UPD of chromosomes 6, 7, 11, 14 and 15 are associated with specific IDs (Transient neonatal diabetes mellitus, Silver-Russell syndrome, Beckwith-Wiedemann syndrome (BWS), upd(14)-syndromes, Prader-Willi syndrome, Angelman Syndrome), the other autosomes are not. UPD of the whole genome is not consistent with life, in case of non-mosaic genome-wide paternal UPD (patUPD) it leads to hydatidiform mole. In contrast, mosaic genome-wide patUPD might be compatible with life. Here we present a 19-year-old woman with BWS features and initially diagnosed to be carrier of a mosaic patUPD of chromosome 11p15. However, the patient presented further clinical findings not typically associated with BWS, including nesidioblastosis, fibroadenoma, hamartoma of the liver, hypoglycaemia and ovarian steroid cell tumour. Additional molecular investigations revealed a mosaic genome-wide patUPD. So far, only nine cases with mosaic genome-wide patUPD and similar clinical findings have been reported, but these patients were nearly almost diagnosed in early childhood. Summarising the data from the literature and those from our patient, it can be concluded that the mosaic genome-wide patUPD (also known as androgenic/biparental mosaicism) might explain unusual BWS phenotypes. Thus, these findings emphasise the need for multilocus testing in IDs to efficiently detect cases with disturbances affecting more than one chromosome.

European Journal of Human Genetics (2013) 21, 788-791; doi:10.1038/ejhg.2012.259; published online 28 November 2012

Keywords: Beckwith-Wiedemann syndrome; cancer; genomic imprinting

\section{INTRODUCTION}

Non-mosaic genome-wide paternal uniparental disomy (patUPD) is commonly known to be lethal. It is usually associated with complete hydatidifrom mole, a condition characterised by the hydropic degeneration of villi and absence of the embryo. In contrast to non-mosaic genome-wide patUPD, its mosaic status-also known as androgenic/biparental mosaicism-is compatible with life and has been reported for several times (Table 1$).{ }^{1-7}$ For non-mosaic genomewide patUPD, different formation mechanisms have been suggested, which mainly occur from prefertilisation stages (for review, refer Van den Veyver and Al-Hussaini ${ }^{8}$ and Golubovsky ${ }^{9}$ ). Mosaic genome-wide patUPD is probably the result of a normal conception followed by post-zygotic errors. ${ }^{10}$

Mosaic genome-wide patUPD carriers show a broad phenotypic range but in all patients Beckwith-Wiedemann syndrome (BWS) and/ or Wilms tumour (WT) were the initial clinical diagnoses (Table 1). In both BWS and WT, (epi)genetic alterations within 11p15.5 can be detected, including paternal UPD 11p15 (upd(11p15)pat)(for review, refer Choufani et $a l^{11}$ ). BWS was initially called EMG syndrome from its three main features exomphalos, macroglossia and (neonatal) gigantism. Additional signs include neonatal hypoglycaemia, hemihypertrophy, organomegaly, earlobe creases, polyhydramnion, haemangioma and cardiomyopathy. In $5-7 \%$ of children, embryonal tumours (most commonly WT) are diagnosed. In addition to the aforementioned BWS features, single genome-wide patUPD carriers present symptoms overlapping with those of other imprinting disorders. ${ }^{6,7}$ As aforementioned, the BWS phenotype does not only comprise dysmorphic and metabolic features, but is also associated with a significantly increased risk for embryonal tumours. As a result, the majority of the genome-wide patUPD patients develop BWSassociated cancers, such as WT or hepatoblastoma.

\section{Case report}

We report on a patient referred to genetic counselling because of BWS features. Her non-consanguineous parents were healthy. The patient has two healthy older brothers and a healthy younger sister. The mother had one miscarriage. Patients and parental chromosomes were normal.

The patient was spontaneously born at 37 gestational weeks. Polyhydramnion was reported. During pregnancy, the mother showed a severe weight gain, but maternal diabetes was not diagnosed. The newborns length was $52 \mathrm{~cm}$ (>P90), weight $3850 \mathrm{~g}$ ( > P90) and head circumference $34,5 \mathrm{~cm}$ (P75-P90). The placenta was reported to be hypertrophic $(2000 \mathrm{~g})$, the umbilical cord was thick $(2 \times 2 \mathrm{~cm}$ at the

${ }^{1}$ Institute of Human Genetics, RWTH Aachen, Aachen, Germany; ${ }^{2}$ Gynaecological Hospital, Department of Gynaecology, Ehingen, Germany; ${ }^{3}$ Praxis für Humangenetik, Ulm, Germany

*Correspondence: Professor T Eggermann, Institute of Human Genetics, RWTH Aachen Pauwelsstr. 30, D-52074 Aachen, Germany. Tel: +49 2418088008 Fax: +49 241 8082394; E-mail: teggermann@ukaachen.de

Received 19 June 2012; revised 6 September 2012; accepted 25 October 2012; published online 28 November 2012 


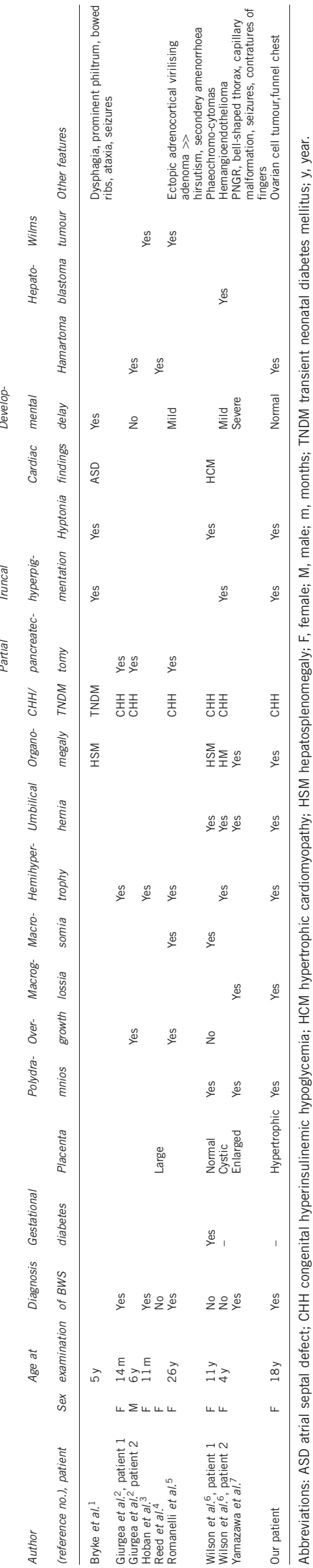

basis). The newborn showed severe postnatal hypoglycaemia with cerebral seizures. Organomegaly was not reported at that time, but a cardiac hypertrophy of the septum and the left ventricle was diagnosed.

At the age of 1 month, a liver hamartoma was resected but there was no evidence for a hepatoblastoma. At 6 weeks, the girl was referred to hospital where hyperinsulinemia and pancreas hypertrophy were diagnosed; parts of the pancreas were resected due to a nesidioblastosis. At the same time, a non-obliterating thrombosis of the Vena cava inferior was observed. Multiple fractures were reported.

Computer tomography at the age of 4 months revealed that the pancreatic head and both kidneys were enlarged. Histology of kidney tissue showed a normal structure. At the same time, a tachypnea was diagnosed and successfully treated.

BWS was diagnosed at the age of 8 months because the girl showed earlobe creases, discrete macroglossia, partial hemihypertrophy of the left body side, an omphalocele, hepato- and nephromegaly.

Because of the unusual clinical observations in our patient, tumour surveillance was continued beyond the age of 10 years, and at the age of 12 years, an enlarged right ovary was detected. At the age of 14 years, the patient developed hypertrichosis and a deep voice. The tumour was resected, pathohistological analyses revealed a steroid cell tumour. Endocrine tests showed an increased level of insulin, whereas other hormones were normal.

At the age of 18 years, the physical examination again confirmed BWS features: in addition to macroglossia and hemihyperplasia, the patients face was characterised by a high forehead and a long face. A funnel chest was reported. Height $(164 \mathrm{~cm})$ and weight were noted as appropriate for age. The patient reported on fibroadenoma of the breast. Hypoglycaemia and hyperinsulinemia persisted during all the time but were well treated. Motoric development was slightly retarded but then appropriate for her age. The patient finished her high-school exam and is currently training as a dental technician.

\section{MATERIALS AND METHODS}

Genomic DNA was extracted by standard techniques from peripheral blood lymphocytes as well as from paraffin-embedded ovarian tumour and breast fibroadenoma tissues, respectively. Because of the initial clinical diagnosis of BWS, a methylation-specific multiplex-ligation-dependent probe amplification assay (MS-MLPA) aiming on the detection of molecular defects in 11p15 (H19 DMR/ICR1, KvDMR/ICR2) was carried out for molecular molecular conformation using a commercially available kit (ME030BWS/RSS, MRC Holland, Amsterdam, the Netherlands). To identify aberrant methylation at further imprinted loci, methylation-specific single nucleotide primer extension (MS-SNuPE) assays were performed (Figure 1a). ${ }^{12}$ To confirm UPD, five microsatellite markers on chromosome 11 and 40 markers on other chromosomes ( $\sim 2$ markers/chromosome) were analysed in DNA from peripheral blood lymphocytes obtained from the patient and her parents (Supplementary Table 1). Seven out of these markers were also typed in DNA from ovarian cancer material and breast tissue according to standard procedures (Figure $1 \mathrm{~b}$ ).

\section{RESULTS}

By initial routine MS-MLPA approaches, we detected both hypermethylation of ICR1 and hypomethylation of the ICR2 in peripheral blood lymphocytes, a finding consistent with upd(11p)pat. Typing of microsatellite markers in $11 \mathrm{p} 15$ additionally confirmed the clinical diagnosis of BWS.

Further MS-SNuPE tests were subsequently performed due to the unusual phenotype. These analyses indeed revealed a disturbed methylation at all eight tested loci (Figure 1a) and indicated that always the paternal alleles were affected. We then performed 


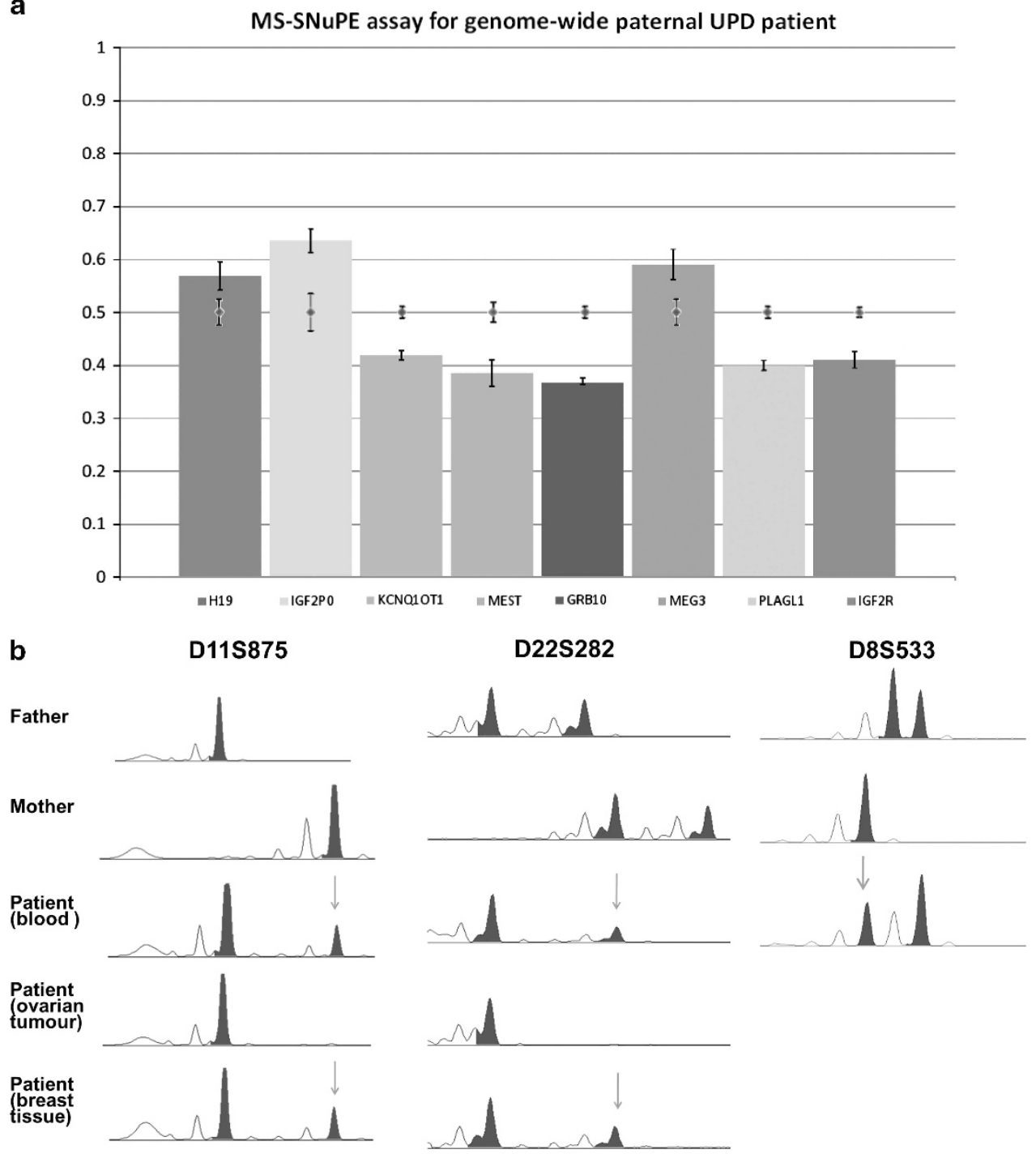

Figure 1 Molecular studies in the patient with genome-wide patUPD. (a) MS-SNuPE results for eight imprinted loci, for each locus two different CpGs were tested. The paternally methylated loci H19, IGF2PO and MEG3 loci showed an increased methylation (nMI>0.5). In contrast a reduced methylation index $(\mathrm{nMI}<0.5)$ can be observed for the maternally methylated loci, PLAGL1, IGF2R, GRB1O, MEST and KCNQ1OT1 corresponding to a genome-wide patUPD. The control ranges are presented as dots (including SDs) for each locus. (the results of the two different CpG loci and from different bisulfit treatments are summarised and SDs are given; for details, refer Begemann et al. ${ }^{12}$ ) (b) Genotyping results of three microsatellite markers (D11S1758 (11p15), D22S282 (22q13), D8S533 (8q13.1)) in the patient and her parents. Patients' DNA from peripheral blood and breast tissue showed a reduction of the maternal allele (red arrows) and an increased signal of the paternal allele, indicating a mosaic patUPD condition. In ovarian tumour DNA, complete genome-wide patUPD was detectable.

genome-wide microsatellite-typing studies in lymphocyte DNA, and thereby confirmed mosaic paternal UPD for 16 autosomes (Figure1b, Supplementary Table 1), markers on the other 6 autosomes were not informative. All informative markers showed a strongly increased peak height of the paternal allele and a lower intensity of the maternal allele. A third paternal allele corresponding to a paternal uniparental heterodisomy was never observed. In DNA from ovarian cancer, tissue typing of seven markers revealed complete non-mosaic genome-wide patUPD, whereas analysis of breast tissue DNA showed the same mosaic patUPD pattern like DNA from peripheral blood (Figure 1b).

\section{DISCUSSION}

The clinical findings and course in our patient with mosaic genomewide patUPD illustrates the broad phenotypic range in carriers of this rare molecular disturbance. However, the preponderant clinical signs in this entity are hyperinsulinemia, organomegaly, umbilical hernia and hemihypertrophy (Table 1), features typically associated with BWS and 11p15 aberrations. Interestingly, a clinical overlap with other imprinting disorders has been reported only for two patients in the literature, though a more complex phenotype might be expected due to different loci affected by the aberration ${ }^{1,7}$ The situation is similar to that in multilocus methylation defect (MLMD) carriers where numerous imprinted loci are affected but the chromosome $11 \mathrm{p}$ phenotypes (BWS, Silver-Russell syndrome/ SRS) are dominant (for review, refer Eggermann et $a^{13}$ ). One might therefore assume that the clinical signs in mosaic genome-wide patUPD (and MLMD) are influenced by further factors, such as tissue-specific mosaicism, tissue and development specific level of imprinted gene expression or interaction between imprinted genes/ domains. 
Moreover, mosaic genome-wide UPDpat is associated with a significantly increased predisposition for cancer development. Five of the so far described 10 patients showed paediatric tumours (pheochromocytoma, hepatoblastoma, pancreatic tumour, adrenocortical neoplasia) (Table 1). This finding is in agreement with the observation that UPD is generally important as a molecular alteration associated with inactivation of tumour-suppressor genes or activation of oncogenes (for review, refer Tuna et al ${ }^{14}$ ) Han et al ${ }^{15}$ recently suggested that the loss of H19 expression is involved in the aetiology of WT. Leaned on the pathomechanism in WT, we hypothesise that genome-wide patUPD leads a decreased expression and thereby influences ovarian tumorigenesis in our patient, ${ }^{16}$ as the ovary is one of the few tissues with $H 19$ expression, which is possibly related to steroid metabolism. On the other hand, it can be assumed that the genome-wide patUPD increases the expression of the paternally expressed insulin-like growth factor 2 (IGF2). ${ }^{17}$ Additionally, the influence of other imprinted genes in ovarian tumorigenesis is conceivable.

The presence of only one paternal allele and the lack of a second paternal allele in all analysed markers excludes prezygotic mechanisms of mosaic genome-wide patUPD formation in our case. We therefore suggest a post-zygotic division error: In an early embryonic stage of a normal biparental diploid zygote, a replication error affecting the maternal DNA followed by endoreduplication of the paternal genome resulted in formation of a normal (biparental) and a uniparental cell line formation. ${ }^{10}$

The identification of mosaic genome-wide patUPD patients has an important impact on genetic counselling and clinical oncology. Considering the clinical course in our patient, we emphasise the importance of further molecular investigations in case of unusual BWS phenotypes. The current genetic BWS testing algorithms are focused on the $11 \mathrm{p} 15$ loci, and dependent on the informativity of the applied tests many mosaic genome-wide patUPD still remain undiagnosed. Furthermore, genome-wide UPD should also be considered in patients with genetic syndromes associated with tumours, as they have a high risk for further neoplasias. In case of BWS, it has been suggested to screen patients for abdominal tumours until the age of 8 years. ${ }^{18}$ Our case now shows that general tumour surveillance is indicated for the whole life in the subgroup of BWS patients with genome-wide patUPD and unusual phenotypes. Furthermore, testing for genome-wide patUPD is generally indicated in BWS patients with upd(11p15)pat.

\section{CONFLICT OF INTEREST}

The authors declare no conflict of interest.

\section{ACKNOWLEDGEMENTS}

The project was supported by the Bundesministerium für Bildung und Forschung (Network 'Imprinting Diseases', 01GM0884) and a scholarship of the German exchange service (DAAD) to MG.

1 Bryke CR, Garber AT, Israel J: Evolution of a complex phenotype in a unique patient with a paternal uniparental disomy for every chromosome cell line and a normal biparental inheritance cell line. Am J Hum Genet 2004 (abstract).

2 Giurgea I, Sanlaville D, Fournet JC et al: Congenital hyperinsulinism and mosaic abnormalities of the ploidy. J Med Genet 2006; 43: 248-254.

3 Hoban PR, Heighway J, White GR et al: Genome-wide loss of maternal alleles in a nephrogenic rest and Wilms' tumour from a BWS patient. Hum Genet 1995; 95: 651-656.

4 Reed RC, Beischel L, Schoof J, Johnson J, Raff ML, Kapur RP: Androgenetic/ biparental mosaicism in an infant with hepatic mesenchymal hamartoma and placental mesenchymal dysplasia. Pediatr Dev Pathol 2008; 11: 377-383.

5 Romanelli V, Nevado J, Fraga M et al: Constitutional mosaic genome-wide uniparental disomy due to diploidisation: an unusual cancer-predisposing mechanism. J Med Genet 2011; 48: 212-216.

6 Wilson M, Peters G, Bennetts B et al: The clinical phenotype of mosaicism for genomewide paternal uniparental disomy: two new reports. Am J Med Genet A 2008; 146A: 137-148.

7 Yamazawa K, Nakabayashi K, Matsuoka K et al: Androgenetic/biparental mosaicism in a girl with Beckwith-Wiedemann syndrome-like and upd(14)pat-like phenotypes. J Hum Genet 2011; 56: 91-93.

8 Van den Veyver IB, AI-Hussaini TK: Biparental hydatidiform moles: a maternal effect mutation affecting imprinting in the offspring. Hum Reprod Update 2006; 12 : 233-242.

9 Golubovsky MD: Postzygotic diploidization of triploids as a source of unusual cases of mosaicism, chimerism and twinning. Hum Reprod 2003; 18: 236-242.

10 Kaiser-Rogers KA, McFadden DE, Livasy CA et al: Androgenetic/biparental mosaicism causes placental mesenchymal dysplasia. J Med Genet 2006; 43: 187-192.

11 Choufani S, Shuman C, Weksberg R: Beckwith-Wiedemann syndrome. Am J Med Genet C Semin Med Genet 2010; 154C: 343-354.

12 Begemann M, Leisten I, Soellner L, Zerres K, Eggermann T, Spengler S: Use of multilocus methylation-specific single nucleotide primer extension (MS-SNuPE) technology in diagnostic testing for human imprinted loci. Epigenetics 2012; 7: 473-481.

13 Eggermann T, Leisten I, Binder G, Begemann M, Spengler S: Disturbed methylation at multiple imprinted loci: an increasing observation in imprinting disorders. Epigenomics 2011; 3: 625-637.

14 Tuna M, Knuutila S, Mills GB: Uniparental disomy in cancer. Trends Mol Med 2009; 15: $120-128$.

15 Han DK, Khaing ZZ, Pollock RA, Haudenschild CC, Liau G: H19, a marker of developmental transition, is reexpressed in human atherosclerotic plaques and is regulated by the insulin family of growth factors in cultured rabbit smooth muscle cells. J Clin Invest 1996; 97: 1276-1285.

16 Ariel I, Weinstein D, Voutilainen R et al: Genomic imprinting and the endometria cycle. The expression of the imprinted gene $\mathrm{H} 19$ in the human female reproductive organs. Diagn Mol Pathol 1997; 6: 17-25.

17 Yun K, Fukumoto M, Jinno Y: Monoallelic expression of the insulin-like growth factor-2 gene in ovarian cancer. Am J Pathol 1996; 148: 1081-1087.

18 Shuman C, Beckwith JB, Smith AC, Weksberg R: Beckwith-Wiedemann syndrome; in Pagon RA, Bird TD, Dolan CR, Stephens K (eds): GeneReviews. Seattle (WA): University of Washington, Seattle, 1993-2000.

Supplementary Information accompanies the paper on European Journal of Human Genetics website (http://www.nature.com/ejhg) 